\title{
An Improved Catastrophic Genetic Algorithm and Its Application in Reactive Power Optimization
}

\author{
Ouyang Sen \\ College of Electric Power, South China University of Technology \\ Guangzhou, China \\ E-mail: OuyangS@scut.edu.cn \\ Received April 22, 2010; revised June 19, 2010; accepted August 17, 2010
}

\begin{abstract}
This paper presents an Improved Catastrophic Genetic Algorithm (ICGA) for optimal reactive power optimization. Firstly, a new catastrophic operator to enhance the genetic algorithms' convergence stability is proposed. Then, a new probability algorithm of crossover depending on the number of generations, and a new probability algorithm of mutation depending on the fitness value are designed to solving the main conflict of the convergent speed with the global astringency. In these ways, the ICGA can prevent premature convergence and instability of genetic-catastrophic algorithms (GCA). Finally, the ICGA is applied for power system reactive power optimization and evaluated on the IEEE 14-bus power system, and the application results show that the proposed method is suitable for reactive power optimization in power system.
\end{abstract}

Keywords: Genetic Algorithms, Reactive Power Optimization, Catastrophe, Power System

\section{Introduction}

The reactive power optimization (RPO), which is a nonlinear planning problem with the traits of discrete, multiple variables and multiple constraints, has a significant influence on secure and economic operation of power systems. It is an effective approach to improve voltage level, decrease network losses and maintain the power system running under normal conditions. In this field, many traditional methods have been used, including linear programming (LP) [1], nonlinear programming (NLP) [2], and interior point methods (IPM) [3]. But, there are some drawbacks in these conventional methods that can limit their application in RPO, such as the complexity of algorithms, poor convergence properties, and the difficulty of dealing with dispersed variables.

Recently, Genetic Algorithm (GA) has been widely used to RPO [4-7]. GA has been theoretically and empirically proven to provide robust search in complex spaces. Numerous papers and dissertations establish the validity of the technique in optimization and control applications [8].

However, it is known that the main shortcoming of GA is premature $[9,10]$. To overcome this, Genetic-Catastrophic Algorithm (GCA) is presented in [11], in which a new operator, catastrophic operator, is proposed to recover the population diversity when premature is happened.
Since the notion of GCA is proposed, there were several researches. Meiying liao [12] analyze the mechanism of catastrophic operator in GA, Yongjun Zhang [13] presented an improved catastrophic operator to enhance the diversity of the small size populations. Others, GCA have been successfully used in many fields of power system, such as RPO [13], optimal power purchase planning [14] and optimal configuration of switching devices in distribution system [15].

The GCA mentioned above is mainly concentrated on improving the searching capability of SGA, but ignoring the instability caused by catastrophic operator. In this paper, based on the analysis of mechanism of GCA, an Improved Catastrophic Genetic Algorithm (ICGA) is proposed. In ICGA, a new catastrophic operator is designed for improving the stability of the proposed method, Besides, in order to improve the GA's convergent speed and searching capability, adaptive genetic algorithm (AGA) is also introduced in this paper, in which crossover and mutation probabilities are varied depending on the number of generations and the fitness value, respectively.

An attempt to apply ICGA to RPO is presented in this paper. The feasibility of the proposed algorithm for RPO is demonstrated and compared with IEEE 14-bus systems. The experimental results prove that the algorithm is reasonable and practicable. 
This paper is organized as follows. Section 2 presents brief reviews on Simple Genetic Algorithm (SGA), and GCA, in Section 3, the ICGA method and the design of crossover and mutation probabilities are proposed respectively. In Section 4, a comparison among the ICGA, SGA and AGA by using typical test function is conducted. Then, the OPR model is mentioned in Section 5, and the results of the simulation for IEEE 14-bus systems are presented in Section 6. Finally a conclusion is presented in Section 7, and the end is references.

\section{Genetic-Catastrophic Algorithm}

GA, first proposed and investigated by John Holland in 1975 [16], is a robust probabilistic search and optimization techniques based on the natural selection and genetic production mechanism. Most of GA works are based on the Goldberg's simple genetic algorithm (SGA) framework [17]. Typically, the process of SGA follows this pattern [18]:

1) Initialization: Generate a first generation $N_{p}$ with random parameters.

2) Evaluate all individuals of the generation $N_{p}$

3) Selection: selects those offspring individuals with a higher fitness value for the next generation $N_{s}$.

4) Crossover: crosses the selected best individuals together to get the new generation $N_{c}$.

5) Mutation: make random mutates of the $N_{c}$, and gets new generation $N_{m}$.

6) Evaluate the individuals of the new generation $N_{p}$, and then go back to 3 ), until certain criteria (such as a fixed number of generations. Or a time) are met.

The main difficulty of application of SGA in engi- neering problems is premature, which occurs due to loss of the population diversity. In order to avoid this problem and improve the convergence properties of SGA, GCA is presented on the based of SGA. In GCA, when premature is happened, catastrophic operator is employed to regain the population diversity.

The process of GCA follows this pattern [11].

1) Initialization

2) Evaluation

3) Selection, Crossover and Mutation

4) If premature convergence occurs, apply catastrophic operator, otherwise go to the step 5)

5) If the convergence criterion is satisfied, terminate the calculation. If not, go to step 2).

The catastrophic operator includes catastrophic condition and operation, catastrophic condition is to judge when to execute catastrophic operation. Generally, catastrophic operation includes two steps:

1) Remove a certain number $\left(N_{c}\right)$ of individuals with low fitness value in current population

2) Regenerate $N_{c}$ individuals via various methods and put into the current population to replace the removed ones.

And the flow chart is shown in Figure 1.

Obviously, by adding various new individuals to the current population, the diversity of population can greatly improve after employing catastrophic operator.

\section{Improved Catastrophic Genetic Algorithm}

Although GCA is an effective method to overcome pre-

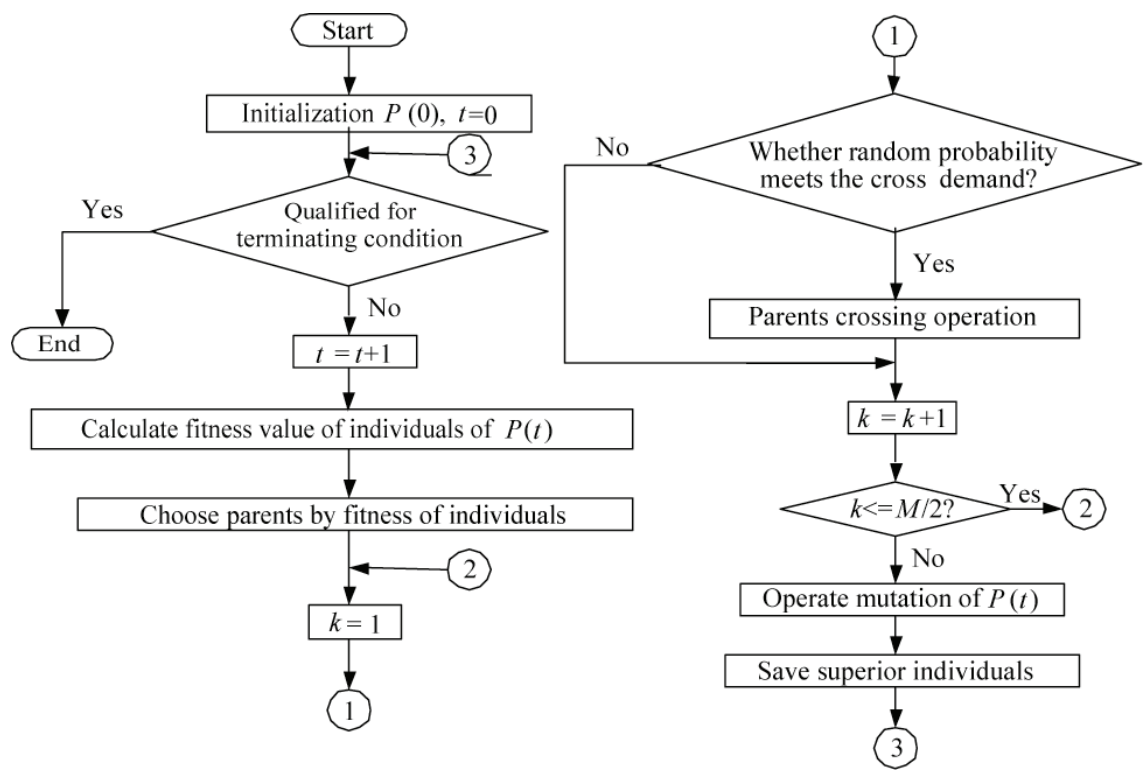

Figure 1. Flow chart of SGA. 
mature, the effect of catastrophic operator on GA's convergence properties is seldom researched. The following subsections will discuss this in more detail.

\subsection{Effect of Catastrophic Operator on GA's Convergence Properties}

\subsubsection{Effect of Traditional Catastrophic Condition on GA's Convergence Properties}

Catastrophic condition is to decide when to execute catastrophic operation, Traditional catastrophic condition is mainly based on the judge of premature convergence $[11,15]$, this method has following disadvantages:

1) Cause disruption of convergence because successful convergence can still meet the catastrophic conditions.

2) Cause overly execution of catastrophic operation because the condition is easily meet, it will bring about the great increment of computing time.

\subsubsection{Effect of Traditional Catastrophic Operation on GA's Convergence Properties}

The key point of catastrophic operation contains two aspects: give an appropriate value for $N_{0}$ and decide how to generate new individuals, in traditional catastrophic operation, $N_{0}$ is a constant value and the new individuals are generated in these ways:

1) Sharply increasing mutation probability $p_{m}$.

2) Remove most of the individuals except the fittest one and randomly generate the new individuals.

These methods can critically affect the convergence performance of GA:

1) Increase the randomness of GA search because the new individuals are separated into the whole solution space.

2) Prolong the convergence time because there are many poor individuals in the new population, which can disrupt the previous superior individuals when they are selected to cross.

3) Disrupt the stability of GA at the late stage of evolution because N0 is a constant value through whole evolution process.

\subsection{The Proposed Algorithm}

In the previous subsection, it is saw that the traditional catastrophic operator can disrupt the GA's convergence properties, and increase the randomness of GA due to inappropriate design of catastrophic operator. In this subsection, the analysis of relationship between the evolution process and GA evolution process will be firstly given. Then, the design of proposed method is presented.

\subsubsection{Analysis of Evolution Process and GA's Convergence Properties}

In the whole GA evolution process, there are tradeoffs that occur in exploration and exploitation. At the early stage of evolution, to explore different solution space and avoid premature, more emphasis should be put on the exploration than exploitation. During the evolution, the population will gradually converge to an optimum solution. At the late stage of evolution, increase exploitation while decrease exploration will allow the GA to prevent the converged optimum solution from disrupting.

As to catastrophic operator, the number of new generated individuals $\left(N_{c}\right)$ is the source of exploration, Setting $N_{c}$ low allows the algorithm to exploit the superior individuals. Setting $N_{c}$ high allows the algorithm to explore different solution space.

\subsubsection{Design of the Proposed Method}

Above analysis inspire us that the convergence properties of GA can be improved by varied $N_{c}$ according to the number of generation. Therefore, $N_{c}$ is given as:

$$
N_{c}=\text { Integer }\left[\exp \left(-a \times t / T_{G e n}\right) \times N_{0}\right]
$$

Where Integer [] is a rounding sign, $t$ is current generation, $a$ is controlled parameter, $T_{G e n}$ is maximum generation, $N_{0}$ is maximum number of new generated individuals.

From the Equation (1), $N_{c}$ is decreased by the number of generation, it means that the exploration is also decreased by the number of generation while the exploitation is on the opposite side. By this way, not only the search ability but also the convergence properties of GA can be enhanced,

The design of catastrophic condition and operation is in the following details:

1) Catastrophic condition:

If $\operatorname{residual}(t / T)=0 \& \& t \leq T$, execute catastrophic operation, where $T$ is the generation steps.

2) Catastrophic operation:

Catastrophic operation is performed by the following steps:

- Save the best individuals in current population

- Remove $N_{c}$ individuals in current population

- Randomly generate $N_{c}$ new individuals

- Add the new generated individuals to the current population.

\subsection{Design of Adaptive Crossover Probability Mutation Probability}

The choice of $p_{c}$ and $p_{m}$ is known to significantly affect the performance of the GA, the $p_{c}$ controls the rate at which solutions are subjected to crossover, the higher the 
value of $p_{c}$ the quicker are the new individuals introduced into the population, vice versa. When a population converges to a global optimal solution (or even a local optimal solution), $p_{c}$ and $p_{m}$ increase and may cause the disruption of the near-optimal solutions, therefore, it is hoped that $p_{c}$ is given a high value to create more individuals at the early stage of evolution and maintaining a relatively low value for the purpose of protecting superior individuals and algorithm's stability at the late stage of evolution process, in this paper, $p_{c}$ is gradually decreased depending on the number of generation in sigmoid function form:

$$
P_{c}(t)=\frac{\left(P_{c \max }-P_{c \min }\right)}{1+\exp \left(\frac{-A \times\left(T_{G e n}-2 t\right)}{T_{G e n}}\right)}+P_{c \text { min }}
$$

where $p_{c}(t)$ is crossover probability generation in $t$ generation, $P_{c \max }$ is maximum crossover probability, $P_{c \max }$ is minimum crossover probability and $A$ is assigned 9.903438 .

Mutation operator is used to maintain genetic diversity from one generation of a population of chromosomes to the next, the choice of $p_{m}$ is critical to GA performance, large values of $p_{m}$ transform the GA into a purely random search algorithm, In this paper, $p_{m}$ is varied adaptively depended on both the number generation and every fitness value of individual:

$$
P_{m t}=\exp \left(\frac{f_{\max }-f\left(X_{i}\right)}{f_{\max }}\right) \times \frac{\left(P_{m \max }-P_{m \min }\right)}{1+\exp \left(\frac{-A \times\left(T_{G e n}-2 t\right)}{T_{G e n}}\right)}+P_{m \text { min }}
$$

where $P_{m}(t)$ is mutation probability of individual $X_{i}$ in $t$ generation, $P_{\max }$ is maximum mutation probability, $P_{\operatorname{mmax}}$ is minimum mutation probability, $A$ is assigned 9.903438 .

\section{Performance Measures}

In this section, the experiments that are conducted to compare the performance of the ICGA, SGA and AGA [19] are discussed. In order to test these methods' convergent speed, the average number of generations that these GA methods require to generate a solution with a certain high fitness value and the average consumed times are taken into consideration. These can be obtained by performing the experiment repeatedly (in our case, 100 times). To measure the convergence probability of these methods, the number of instance (in 100 trials) for which the GAs have successfully converged to the given optimal solution is also employed.

\subsection{Test Functions}

In this research, the following multimodal functions with varying complexities are used [19]:

$$
\begin{gathered}
\left\{\begin{array}{l}
f_{1}\left(x_{1}, x_{2}\right)=100\left(x_{1}^{2}-x_{2}\right)^{2}-\left(1-x_{1}\right)^{2} \\
-2.048 \leq x_{i} \leq 2.048 \quad(i=1,2)
\end{array}\right. \\
\left\{\begin{array}{l}
f_{2}\left(x_{1}, x_{2}\right)=0.5+\frac{\sin ^{2} \sqrt{x_{1}^{2}+x_{2}^{2}}}{\left[1.0+0.001\left(x_{1}^{2}+x_{2}^{2}\right)\right]^{2}} \\
-100 \leq x_{i} \leq 100 \quad(i=1,2)
\end{array}\right.
\end{gathered}
$$

Function $f 1$ is a two-dimension function and has only one minimum in its whole solution space. But it is an abnormal function and is not optimized easily. Its global minimum is $f 1(1,1))=0$; Function $f 2$ is a rapidly varying multimodal function with two variables, and is symmetric about the origin with the height of the barrier between innumerable adjacent minima increasing as the global optimal solution is approached. $f(0,0)=0$ is its global minimum

\subsection{Experiment Results}

In all our experiments, the population size for all functions is 64 and $T_{G e n}$ is given 100, For the SGA, $P_{c}=0.9$, $P_{m}=0.1$, For the AGA, $k 1=k 3=1, k 2=k 4=0.5, P_{c \max }$ $=0.9, P_{c m i n}=0.5, P_{m m a} x=0.1, P_{\text {mmin }}=0.01$. And for the ICGA, $P_{c \max }=0.9, P_{c \text { min }}=0.5, P_{\text {mma }} x=0.1, P_{\text {mmin }}=0.01$, $a=10, N 0=59$. The algorithm will be stop when each GAs attaining a solution with an objective functional value of $f 1(f 2)$ equal to the threshold 0.0001 , the experimental results are presented in Table 1:

As Table 1, both average generation and consumed time of ICGA are less than SGA and AGA, Others, the convergence times of ICGA is the best, it indicates that ICGA can effectively 'jump' from the local optimum.

\section{Reactive Power Optimization Model}

RPO is mainly implemented by setting generator bus voltages, VAR compensators and transformer taps. The core of the RPO problem is to determine the reasonable reactive power compensation point and the best compensation capacity, than it can minimize the real power losses and improve voltage profile. The models of reactive power optimization are described as follows:

\subsection{Objective Function}

$$
P_{l o s s}=\sum_{i=1}^{N} V_{j} \sum_{j \in h} V_{j}\left(G_{i j} \cos \delta_{i j}+B_{i j} \sin \delta_{i j}\right)
$$


Table 1. Comparison of performance of SGA, AGA and ICGA.

\begin{tabular}{cccccccccc}
\hline Function & \multicolumn{3}{c}{ Average Generations } & \multicolumn{3}{c}{ Convergence Times } & \multicolumn{3}{c}{ Consumed Time } \\
\hline$f 1$ & SGA 91 & AGA 61 & ICGA 41 & SGA 82 & AGA 92 & ICGA 97 & SGA 0.47 & AGA0.36 & ICGA 0.28 \\
$f 2$ & 48 & 35 & 28.9 & 71 & 91 & 99 & 0.39 & 0.32 & 0.22 \\
\hline
\end{tabular}

where $P_{\text {loss }}$ is net loss for meritorious indicators, $N$ is the number assemble of transmission lines in the network, $V_{i}$ is the voltage value at bus $i, V_{j}$ is the voltage value at bus $j, \delta_{i j}$ is the phase-angle difference of the voltage value between bus $i$ and $j, G_{i j}$ and $B_{i j}$ are admittance matrix elements in the real and imaginary parts.

\subsection{Constraints}

$$
\left\{\begin{array}{l}
P_{i}=V_{i} \sum_{j=1}^{N} V_{j}\left(G_{i j} \cos d_{i j}+B_{i j} \sin d_{i j}\right) \\
Q_{i}=V_{i} \sum_{j=1}^{N} V_{j}\left(G_{i j} \sin d_{i j}-B_{i j} \cos d_{i j}\right)
\end{array}\right.
$$

where $P_{i}, Q_{i}, V_{i}$ is active power, reactive power and voltage of node $i$ respectively.

Variable equation:

Power system safe operation must be made within the scope. Select capacitive reactive power compensation capacity $C_{i}$, adjustable transformer tap position $T_{j}$ and generator terminal voltage $V_{g k}$ as the control variables. And select and Node voltage amplitude $V i$ as state variables reactive power generators $Q_{g j}$.

$$
\left\{\begin{array}{l}
C_{i \min }<C_{i}<C_{i \max } \\
T_{j \min }<T_{j}<T_{j \max } \\
V_{g k \min }<V_{g k}<V_{g k \max }
\end{array}\right.
$$

The unequal constraint (9) is the unequal constraint of the control variable vector, where $T_{\text {jmin }}, T_{\text {imax }}$ is adjustable load transformer tap position of the upper and lower limit. $C_{\text {imin }}, C_{\text {imax }}$ is capacitive reactive power compensation capacity of the upper and lower limit; $V_{g k m i n}, V_{g k m a x}$ is generator terminal voltage the upper and lower limits.

$$
\left\{\begin{array}{l}
V_{i \min }<V_{i}<V_{i \max } \\
Q_{g j \text { min }}<Q_{g j}<Q_{g j \text { max }}
\end{array}\right.
$$

The unequal constraint (10) is the unequal constraint of dependent variable vector, where $V_{\text {imin }}, V_{\text {imax }}$ are the node voltage amplitude of the upper and lower limit. $Q_{\text {gimin }}, Q_{\text {gimax }}$ are the limits of generators reactive power. The control variables are self-constrained and dependent variables are constrained by adding them as penalty terms to the objective function (Equation (1)). So the objective function is changed to:

$$
\min P_{\text {loss }}+\lambda_{V} \sum\left(\frac{\Delta V_{i}}{V_{i \max }-V_{i \min }}\right)^{2}+\lambda_{Q} \sum\left(\frac{\Delta Q_{g i}}{Q_{g i \max }-Q_{g i \min }}\right)^{2}
$$

where $\lambda_{V}$ and $\lambda_{Q}$ are penalty factors; $\Delta V_{i}$ and $\Delta Q_{g i}$ are the violations of load-bus voltages and generators reactive power, respectively, defined as:

$$
\begin{gathered}
\Delta V_{i}= \begin{cases}V_{i \min }-V_{i} \quad V_{i}<V_{i \min } \\
0 \quad V_{i \min } \leq V_{i} \leq V_{i \max } \\
V_{i}-V_{i \max } & V_{i}<V_{i \max }\end{cases} \\
\Delta Q_{g i}=\left\{\begin{array}{cc}
Q_{g i \min }-Q_{g i} & Q_{g i}<Q_{g i \min } \\
0 & Q_{g i \min } \leq Q_{g i} \leq Q_{g i \max } \\
Q_{g i}-Q_{g i i \max } & Q_{g i}<Q_{g i \max }
\end{array}\right.
\end{gathered}
$$

\section{Numerical Results}

The study is implemented using Matlab and tested on an IEEE 14-bus system, which includes three transformers, two generators and three compensation points. The step size of reactive power compensation and transformer tap ratios is $0.05 \mathrm{pu}$ and $0.0125 \mathrm{pu}$ respectively. Capacity ceiling of Reactive compensation device is $0.5 \mathrm{pu}$, and the step is $0.01 \mathrm{pu}$. The control variables are showed as: $Y=\left[\begin{array}{llllllll}U_{G 1} & U_{G 2} & Q_{G 3} & Q_{G 6} & Q_{G 8} & T_{47} & T_{49} & T_{56}\end{array}\right]$. Details of this system and parameters are given in [20]. The number of generation is 30 and other parameters are following: $T_{G e n}$ $=30, N_{0}=29, \lambda_{V}=15, \lambda_{Q}=1.5$. The best optimization results of SGA, ICGA and GCA are given in Table 2, which are obtained from different kinds of algorithm respectively performing 30 times.

As Table 2, the initial generator reactive power $Q_{G 2}$ and load voltages $V_{D 3} \sim V_{D 14}$ are outside their limits, after optimization by the three methods, all the state variables are regulated back into their limits, besides, $P_{\text {loss }}$ is decreased to 0.1375 by ICGA while that of 0.1378 by GCA and 0.1385 by SGA, thus, ICGA may find better outcome than the rest kinds of methods.

Table 3 shows the comparison of the best, worst, average and standard deviation values for different methods. Due to probabilistic characteristic of evolutionary algorithms, results reported here correspond to average from 30 trials. 
Table 2. Variables of IEEE 14-bus system.

\begin{tabular}{|c|c|c|c|c|c|c|}
\hline Var. & Lower limit & Upper limit & Initial Value & SGA Results & GCA Results & ICGA Results \\
\hline$U_{G 1}$ & 0.95 & 1.05 & 1.000 & 1.05 & 1.05 & 1.05 \\
\hline$U_{G 2}$ & 0.95 & 1.05 & 1.000 & 1.0339 & 1.0307 & 1.0371 \\
\hline$T_{47}$ & 0.90 & 1.10 & 0.975 & 1.05 & 0.9875 & 0.9875 \\
\hline$T_{49}$ & 0.90 & 1.10 & 1.000 & 1.000 & 1.075 & 1.075 \\
\hline$T_{56}$ & 0.90 & 1.10 & 0.925 & 1.000 & 1.000 & 1.000 \\
\hline$Q_{G 3}$ & 0.00 & 0.40 & 0 & 0.31 & 0.37 & 0.32 \\
\hline$Q_{G 6}$ & -0.06 & 0.24 & 0 & 0.23 & 0.24 & 0.24 \\
\hline$Q_{G 8}$ & -0.06 & 0.24 & 0 & 0.15 & 0.24 & 0.24 \\
\hline$Q_{G 1}$ & -0.50 & 1.50 & -0.295 & -0.1231 & -0.0816 & -0.2098 \\
\hline$Q_{G 2}$ & -0.50 & 1.00 & 1.515 & 0.5077 & 0.3146 & 0.4939 \\
\hline$U_{D 3}$ & 0.95 & 1.05 & 0.9111 & 0.9983 & 1.0048 & 1.0051 \\
\hline$U_{D 4}$ & 0.95 & 1.05 & 0.9228 & 0.9961 & 1.0014 & 1.0049 \\
\hline$U_{D 5}$ & 0.95 & 1.05 & 0.9295 & 1.0041 & 1.0074 & 1.0109 \\
\hline$U_{D 6}$ & 0.95 & 1.05 & 0.9245 & 1.0037 & 1.0084 & 1.0119 \\
\hline$U_{D 7}$ & 0.95 & 1.05 & 0.9077 & 1.0178 & 1.0071 & 1.0103 \\
\hline$U_{D 8}$ & 0.95 & 1.05 & 0.9077 & 1.0427 & 1.0474 & 1.0499 \\
\hline$U_{D 9}$ & 0.95 & 1.05 & 0.8891 & 0.9903 & 0.9934 & 0.9967 \\
\hline$U_{D 10}$ & 0.95 & 1.05 & 0.8865 & 0.9848 & 0.9881 & 0.9915 \\
\hline$U_{D 11}$ & 0.95 & 1.05 & 0.9011 & 0.9905 & 0.9945 & 0.9979 \\
\hline$U_{D 12}$ & 0.95 & 1.05 & 0.9080 & 0.988 & 0.9926 & 0.9961 \\
\hline$U_{D 13}$ & 0.95 & 1.05 & 0.8989 & 0.983 & 0.9875 & 0.9911 \\
\hline$U_{D 14}$ & 0.95 & 1.05 & 0.8719 & 0.9679 & 0.9716 & 0.9751 \\
\hline$P_{\text {loss }}$ & & & 0.1746 & 0.1385 & 0.1378 & 0.1375 \\
\hline
\end{tabular}

Table 3. Comparison of best, worst, average and standard deviation values for SGA, GCA and ICGA.

\begin{tabular}{ccccc}
\hline & $\begin{array}{c}\text { Best } \\
\text { Ploss/pu }\end{array}$ & $\begin{array}{c}\text { Worst } \\
\text { Ploss/pu }\end{array}$ & $\begin{array}{c}\text { Average } \\
\text { Ploss/pu }\end{array}$ & $\begin{array}{c}\text { Stardard } \\
\text { Deviation }\end{array}$ \\
\hline SGA & 0.1385 & 0.1500 & 0.1423 & 0.0030 \\
GCA & 0.1780 & 0.1464 & 0.1405 & 0.0019 \\
ICGA & 0.1375 & 0.1394 & 0.1380 & $4.88 \mathrm{E}-04$ \\
\hline
\end{tabular}

As Table 3, the results found by the ICGA are apparently better than those obtained by SGA and GCA. Because standard deviation clearly reflects degree of scatter about simulation results, the result illustrates that ICGA has preferable convergence stability.

\section{Conclusions}

Although Catastrophe Genetic Algorithms is an effective method to enhance GA's global search ability, it comes up with the problem of instability. To solve this conflict, this paper presents the ICGA. In this method, new catastrophic operator is proposed and new adaptive crossover and mutation probability is designed. And the comparison with test functions shows that not only the stability of ICGA is greatly improved, but also the convergent speed and global searching capability. The proposed ICGA is applied to reactive power optimization of power system. The simulation results of the IEEE 14-bus system indicate that ICGA can be able to undertake global search with a fast convergence rate and a feature of pref- 
erable convergence stability. It is proved to be efficient and practical during the reactive power optimization.

\section{References}

[1] K. R. C. Mamandur and R. D. Chenoweth, "Optimal control of reactive power flow for improvements in voltage profiles and for real power loss minimization," IEEE Transaction on PAS, Vol. 100, No. 7, 1981, pp. 31853194.

[2] L. C. Li, J. Y. Wang, L. Y. Chen, et al., "Optimal reactive power planning of electrical power system," Proceedings of the CSEE, Vol. 19, No. 2, 1999, pp. 66-69.

[3] M. B. Liu and X. C. Wang, "An application of interior point method to solution of optimization problems in power systems," Power System Technology, Vol. 23, No. 8, 1999, pp. 61-64, 68

[4] K. J. Iba, "Reactive Power Optimization by Genetic Algorithm," IEEE Transactions on Power System, Vol. 9, No. 2, 1994, pp. 685-692.

[5] P. Nallagownden, L T. Thin and N. C. Guan, "Application of Genetic Algorithm for the Reduction of Reactive Power Losses in Radial Distribution System," IEEE International Conference on Power and Energy, Vol. 26, No. 5, 2006, 76-81.

[6] P. Sreejaya and R. Rejitha, "Reactive power and Voltage Control in Kerala Grid and Optimization of Control Variables Using Genetic Algorithm," IEEE International Conference on Power System Technology, 2008, 1-4.

[7] H. W. Yan and J. N. Tao, "Reactive power optimization research of power system considered the generation transmission and distribution," IEEE International Conference on Industrial Technology, Chengdu, 2008, pp. $1-4$.

[8] D. E. Goldberg and P. Segrest, "Finite Markov chain analysis of genetic algorithms," Proceedings of 2 nd International Conference Genetic Algorithms, 1987, pp. $1-8$.

[9] J. E. Baker, "Reducing bias and inefficiency in the selection algorithm," Proceedings of the 2nd Annual Conference on Genetic Algorithms, Massachusetts Institute of
Technology, Cambridge, 1985, pp. 14-21.

[10] D. E. Goldberg, "Genetic algorithms and rule leaming in dynamic system control," Proceedings of International Conference on Genetic Algorithms and Their Applications, Pittsburgh, 1985, pp. 8-15.

[11] X. D. Jin and Z. Li, "Genetic-Catastrophic Algorithms and Its Application in Nonlinear Control System," Journal of System Simulation, Vol. 9, No. 2, 1997, pp. 111115.

[12] M. Y. Liao and Y. J. Zhang, "Study on the Effect of Cataclysm Operator on Genetic Algorithm," Computer Engineering and Application, Vol. 41, No. 13, 2005, pp. 5456.

[13] Y. J. Zhang, Z. Ren, H. M. Zhong, Z. Y. Tang and C. Shang, "Cataclysmic Genetic Algorithms Based Optimal Reactive Power Planning," Automation of Electric Power Systems, Vol. 26, No. 23, 2002, pp. 29-32.

[14] Y. J. Zhang, W. G. Yuan, B. F. Li and M. C. Liao, “Optimal Power Purchase Planning of Hainan Power Grid Company," The 8th International Power Engineering Conference, December 2007, Singapore, pp. 1854-1858.

[15] D. Wang, Y. K. Shi, J. Y. Cong, H. Sun and J. Y. Zou, "Application of Catastrophic Genetic Algorithm to the Opitmal Configuration of switching Devices in Distribution System," High Voltage Apparatus, Vol. 40, No. 3, 2004, pp. 180-182.

[16] J. H. Holland, "Adaptation in natural and artificial systems," University of Michigan Press, Ann Arbor, 1975.

[17] D. E. Goldberg, "Genetic Algorithms in Search, Optimization and Machine Learning," Addison-Wesley Publishing Company Inc., Massachusetts, 1989.

[18] L. Davis, Ed., "Genetic Algorithms and Simulated Annealing," Pitman, London, 1987.

[19] M. Srinivas and L. M. Patnaik, "Adaptive probabilities of crossover and mutation in genetic algorithm," IEEE Transactions on System, Man and Cybernetics, Vol. 24, No. 4, 1994, pp. 656-667.

[20] Y. J. Zhang, "Cataclysmic Genetic Algorithm and MAS Based Model for Reactive Power Optimization for Power System," Ph.D. Dissertation, South China University of Technology, Guangzhou, 2004. 